\title{
ACCIDENT RISK ASSESSMENT FOR HIGHLY RELIABLE AVIATION SYSTEMS IN EMERGENCY SITUATIONS
}

\author{
Evgeniy Kuklev ${ }^{1}$, Viatcheslav Zhilinsky (Žilinskis) ${ }^{2}$ \\ ${ }^{1}$ St. Petersburg State University of Civil Aviation \\ St. Petersburg, Russian Federation, Pilot str.38 \\ (812)704 25 86; info@spbguga.ru \\ ${ }^{2}$ Baltic International Academy \\ Riga, Latvia, Lomonosov str. 4, LV-1019 \\ (371) 29211 672;v.zhilinsky@gmail.com
}

\begin{abstract}
Safety of aircrafts exploitation is estimated by means of the using the results of the prognoses of the dangerous factors which could be take place during the flight in the off-design conditions. The risk - approach, based on the fuzzy sets methods, is discussed and find out the paths leading to the catastrophes of the different types of the vessels. The dangerous (Unsafeness, Hazard) is the discrete state with the factors which could be take place during the flight in the off-design conditions. It was suggested to find out the paths leading to the catastrophes under condition of the rare events with probabilities "almost zero".
\end{abstract}

Keywords: rare events, risk, civil aviation, flight safety, fuzzy sets

\section{Introduction}

Fundamental for the offered research area, the publications and wide confession of worldwide known sources in the examined area: the standards worked out by the Austrian Institute of Standards for application in the field of solving the issues of safety (Austrian Standards: ONR 49000:2010, 2010) and Riga's effort (Khalkis et al., 2005), including the sphere of the Civil Aviation (CA) and few works of IATA (IATA Safety Report, 2014).

However, for CA it is necessary to do some amendments in a division of "Terms" (ASI, 2010; IATA, 2014; Khalkis et al., 2005) to apply the working methodologies on solving of tasks in the theory of safety flights (SF) taking into account the rare events.

In the "Austrian standards" there are improper determinations of the "risk concept" for the rare events. The rare events arise up in technique as elements with the probability "almost a zero" (ICAO Conference of higher level of Flight Safety, 2010; Everdij et al., 2013; Amer, 2012). Moreover, the one is determined on "the tails" of a density of the probability distribution of the events. Statistics on such events, which are disposed on "the tails", is absent. The mathematical expectations, variances and the probability distribution parameters for the rare events don't exist practically. Finding of the reliable probabilistic estimations of indexes of SF is practically impossible. That is why the different amendments must be done as soon as possible in order that the theory of safety will be become more universal and correct (Amer, 2012).

In the article it is suggested to apply the perspective approach of the Fuzzy Sets methods or minimax criteria (Khalkis et al., 2005) for development of the unconventional risk models of appearances of the rare events as well as the methods of their analysis. The solutions of the methodical difficulties on the definition of the integrated risk level of danger so as the $\hat{\widetilde{R}}$ by NASA (Probabilistic Risk Assessment PRA, 2002), created in Fuzzy Sets (Gipich et al., 2013) as elements of the aviation systems, are considered in the article.

It is necessary to point out that lack of the theory and the concept of risk and the rare events based on the ideas of NASA were of the publications in Riga Civil Aviation Institute of an unsuccessful interpretation the average risk in the form that it is the pure multiplication: "the probability of an event" with "the damage level ". But there are very important theoretical issues when in the situations with the rare events, this position gives the scalar risk value which will be always equal to "ZERO" (Khalkis et al., 2005; IATA Safety Report, 2014), instead of $\hat{\widetilde{R}}$ by NASA (Probabilistic Risk Assessment (PRA), 2002). This question will be complementary to discuss below. 


\section{Background}

The main term "risk" is certain, according to the published works (Malinowsky et al., 2000), as a measure of danger for scenarios resulting in the points "of vulnerability" and "windows of vulnerability". A risk concept cannot be given as "probability", that it will be absurd (Probabilistic Risk Assessment (PRA), 2002; Gipich et al., 2013) as rare events in (Khalkis et al., 2005). Determination of risk is formulated here in accordance with a good sense, for example "on Oxford" (The Little Oxford Thesaurus, 2006). The New classification of intercommunication of the basic terms corresponds it in the systems safety theory (safety, danger, threat, vulnerability etc.), the formula of estimation of integral size of risk is introduced. Some results offered in Civil Aviation can be presented as an example, where norms are given on the classification of ICAO and NASA.

It must be accepted that such a phenomenon as the risk event $\mathrm{R}$ is very rare. Statistics about this one does not exist, but the small data quantities to calculate probabilities are not enough. That is why the traditional probabilistic concept "of risk" as methodical or mathematical category is unacceptable in the problem of rare events and also in the modern theory of the safety systems.

Nancy Levisohn in (2013) has noticed that "application of probabilistic concept "of risk" in the theory of safety is incorrect in the case of the rare events".

This opinion also coincides with the theoretical positions of the Russian specialists in civil aviation. It is the declaration for developing the approach based on the Fuzzy Sets (Gipich et al., 2013), as shown above. In this connection the positions of TSF, mentioned earlier have been accepted here as the follows:

-"Catastrophe" (as the phenomenon and possible event) is included into the system and only waits for the way out (by professor J. Reason from MIT and NASA);

- Negative events (type "of catastrophes", "accidents", serious emergency incidents) - are rare so that statistics on these events is unsteady, (insufficient) values is unknown, and practically cannot be found for certain, the concept of the average time of the catastrophe (serious accidents) does not have a physical sense by comparison to the theory of reliability;

-"Safety is discreet state" (ICAO Conference of higher level of Flight Safety, 2010; Gipich et al., 2013).

\section{Formal description of risk concept on ICAO}

This official description is presented here in a well-known and unclear (Everdij et al., 2013; Amer, 2012; Probabilistic Risk Assessment (PRA), 2002).

Risk Concept: Likelihood (L) \& Severity of Harm (H).

However, the methods of measuring risks and types of the combination of full parameters in this conception are not indicated. There are only the general pointing on the factors $(L)$ and $(H)$ and on qualificatory risk meaningfulness. Generally, the accepted unclear estimations of risk meaningfulness is (4) also not indicated. Nevertheless, they must be. The methods of the evaluation of the risk levels must be considered in the next unclear form (classic - on L. Zadeh, for example, from (The Little Oxford Thesaurus, 2006; Gipich et al., 2013)).

(Risk is large, Risk is small, Risk is not meaningful, etc).

It on (1), (2) is difficult or sometimes impossible because of the vagueness of the risk concept the rare events: therefore, there were the methodical difficulties on the determination of the integral risk level. The overcoming method is discussed in the present paper that allows to expand the theory of system safety (TSS) more consistently.

Formalization of risk event as the rare phenomenon

The similar risk event with uncertainty of the negative consequences (Malinowski et al., 2000) so as harm from a risk event is presented here in the next as follows (Smurov et al., 2012):

$R=R\left(\gamma \mid Z_{R}, \mu, H_{R}, \Sigma_{o}\right)$,

where $Z_{R}$ is a vagueness of the set kind $\gamma$ from a classifier;

$\mu$ is an unclear measure, for example, as a loss of property of functionality i.e. "failures" - on FMEA (ECAST, 2012; Gupich et al., 2013); 
$H_{R}$ is a damage;

$\Sigma_{0}$ are terms of the origin of the near-accident (scenario) conditioned by the threat of $Z_{R}$;

a sign $(\ldots \mid \ldots)$ is a line in (3) which designates the properties of the experiment, giving the event of $R$ entered in consideration taking into account terms characterizing the set experiment.

Problems are that some danger factors, including the following parameters as $(L)$ and $(H)$ in (1) and also $\left(\mu, H_{R}\right)$ are not create the topological space on basis (3). It is more successful to find the mathematical formula of the danger of the situation according to the conception of the risk (and danger) situation (4) in form $\tilde{R}$ (Gupich, 2013; Smurov, et al., 2012) by ICAO (IATA safety report, 2015).

Than the danger situation $\tilde{R}$ will be done as the cortege:

$\tilde{R}=\left\langle Z_{R}, \mu, H_{R} \mid \Sigma_{0}\right\rangle$,

where $\mu$ (or $\mu_{1}$ ) is a vagueness of some kind from a classifier (ICAO Conference of higher level of Flight Safety, 2010) so that is an unclear measure of the danger possibility of the origin of the risk event, for example, as a loss of property of functionality on (ICAO Conference of higher level of Flight Safety, 2010) or it is a damage (Advisory Circular, 2006; Report of GosNII AS, 2010).

In terms of the origin of the situation (4) there is the same symbol as in (3) for the properties of the experiment, giving an event as terms characterizing an integrated value of risk as measures of the unclear estimations of an amount of danger in the set of the states. Thus, these measures correspond to the matrix $M_{R}$ of risk (by ICAO - NASA). It is the basis of indicators dates for the matrix $M_{R}\left(J_{i}, L_{k}\right)$ of NASA (Probabilistic Risk Assessment (PRA), 2002).

Then it may get the algorithm of evaluation of the level of integral risk by means of the matrix of NASA (Probabilistic Risk Assessment (PRA), 2002), that is needed for a preventive management of $\tilde{\widetilde{R}}$, based on monitoring of the hazardous and danger factors in the systems of type of SMS - Safety Management System (IATA Safety report, 2014) at form (with accordance of NASA matrix) as:

$$
\hat{\tilde{R}}=\hat{f}\left(\tilde{R} \mid \Sigma_{0}\right)=\hat{f}\left(Z_{R}, \mu, \mu_{1}, H_{R} \mid \Sigma_{0}\right),
$$

where, $\hat{\widetilde{R}}$ is the total level of risk on fuzzy interpretation (5) by means of the matrix of NASA.

It is preliminary suggested to produce breaking up of formula (6) of ICAO (4) on the components $(L)$ and $(H)$ by the appeal of the procedure, giving the result of this unclear implication (5). Thus, the risk situation by ICAO determined in form (5) of concept of "danger" in the system through the hazard for safety of $Z_{R}$ must be accepted in all the well-known theories (Everdij et al., 2013; Amer, 2012; PRA, 2002). Then the unclear ("verbal") formula (1) of ICAO will be considered in future as that allows to find the size of integral risk $\hat{\widetilde{R}}$ by NASA.

The hazard (or threat) of $Z_{R}$ plugs itself in the set of the factors of dangers, which result in the origin of hazardous risk occurrence (6) on one of the alternative chains of the events to be the catastrophe in the corresponding critical scenario of the events.

The estimation of the scenario danger $\tilde{R}$ proposed in (5) with an account (6) has the appearance in form of cortege with two arguments (minimum) or class of threat of $Z_{R}$ with some sets of the danger factors of $\varphi_{i} \in Z_{R}$ :

$\varphi_{i} \in \Phi=\left\{\varphi_{\mathrm{i}} \mid Z_{R}, \Sigma_{0}\right\}$

Furthermore, with (4), (5) it is succeeded to make the well-known matrix of $M_{R}\left(J_{i}, L_{k}\right)$ for the analysis of risks with the indicators of the possibilities $J_{i}$ and the category of the seriousness of the consequences of $L_{k}$ in the form NASA (IATA Safety Report, 2014). A similar matrix was specially entered in and ratified in order to legalize the application on the basis of (5) the unclear levels of risk in the form indicated above with using (4). 
Then the general risk in case of using the matrix of risks (on NASA, ICAO) will be now a size or value of the risk level the unclear estimations as an amount or measure of danger (by NASA, ICAO). It is the basis of the indicators for the risk levels, finding by means of the matrix, accepted earlier. It provides the solution of the problem of the rare events. An amount or measure of danger is determined here in the form as it is a function from multitude with two arguments so as $\widetilde{R}$ - cortege (3) accepted above in the description of $\hat{\widetilde{R}}$.

The preventive (proactive) managing decisions, recommendable ICAO on the basis of the categories of the events of the type of $R$ in form (3) with their integral quantity the unclear level. Thus, the meaningfulness of the risk situation offers to estimate the one on the basis model (3), (4) of risk. It is here suggested that is existing.

The rare risk event $R$ is as indefinite element of an experiment with the negative consequences so as harm and unclear possibility (with some measure). This similar risk event is given in form (3) for the situation (4).

A parameter of vagueness of a rare event of such a kind as (3) with the measures is taken from the classifier (ICAO Conference of higher level of Flight Safety, 2010). By physical sense of (3), (4) there are here an unclear measure of a possibility of the origin of the risk event, for instance, a loss of property of functionality (ICAO, 2010). For example, it is a damage or also there are the danger factors from hazardous of situation or scenarios of (4) so as in (3).

In further there are here $\Sigma_{0}$ the same conditions of the activities of the systems so such as in (3) (class and model of danger, chain of scenarios, etc).

The critical failures so such as catastrophes in the systems are found upon the method of the minimal sets of the failures by means of the use of a tree of the events (ICAO Conference of higher level of Flight Safety, 2010).

\section{Warning process of risks mitigation in SMS by ICAO}

The general algorithm of NASA for the choice of the correcting affecting on the system has the following expression:

$\{$ Hazards - a risk event - prognosis of scenario of events, leading to the catastrophes a dangerous state - a risk assessment - managing influences $\}$

In (7) proactive prognostication or finding out the really existent source of danger (hazard) is primary. The Unforeseeable appearance of the hazardous occurrence from the unclear subset of some clear universal set ("the carrier") takes place in (5) with some measure of the truth by L. Zadeh. The real level of danger $\hat{\widetilde{R}}$ is easily estimated in future only on the basis of the methodology of the calculations of the risks by use the Fuzzy Sets and matrix $M_{R}\left(J_{i}, L_{k}\right)$ (Fig. 1). The hazards (and factors of danger) effect on the systems through the unclear windows of the vulnerability.

\begin{tabular}{|c|c|c|c|c|c|}
\hline \multirow[b]{2}{*}{ Likelihood } & \multicolumn{5}{|c|}{ Severity of risk } \\
\hline & $\begin{array}{c}\text { Catastrophic } \\
\text { A }\end{array}$ & $\begin{array}{c}\text { Hazardous } \\
\text { B }\end{array}$ & $\underset{\mathrm{C}}{\text { Major }}$ & $\begin{array}{c}\text { Minor } \\
\text { D }\end{array}$ & $\begin{array}{c}\text { No Safety } \\
\text { Effect } \\
\text { E }\end{array}$ \\
\hline Often & $5 \mathrm{~A}$ & 5B & $5 \mathrm{C}$ & $5 \mathrm{DD}$ & $5 \mathrm{E}$ \\
\hline Sometimes & $4 \mathrm{~A}$ & $4 \mathrm{~B}$ & $4 \mathrm{C}$ & 4D & $4 \mathrm{E}$ \\
\hline Quite rarely & $3 \mathrm{~A}$ & $\overline{3 B}$ & $3 \mathrm{C}$ & 3D & $3 \mathrm{E}$ \\
\hline Very rarely & $2 \mathrm{~A}$ & $\overline{2 B}$ & $2 \mathrm{C}$ & $2 \mathrm{D}$ & $2 \mathrm{E}$ \\
\hline $\begin{array}{l}\text { Extremely } \\
\text { rarely }\end{array}$ & 1A & 1B & $1 \mathrm{C}$ & 1D & $1 \mathrm{E}$ \\
\hline
\end{tabular}

Figure 1. The NASA matrix

This level of danger is resulted at methodology of the calculations of the level risks by use the Fuzzy Sets (Gipich et al., 2013). The hazards (and factors of danger) have impact on systems through the fuzzy window of the vulnerability, where the hazards can be come. 


\section{Conclusion}

The risks assessment method of catastrophes appearance which are rare events is based on Fuzzy Sets for tasks of providing the flights safety.

For the systems with the general events space determined on Boolean lattice, it is suggested to apply the use of the fuzzy criteria for verification in form of the "possibility of loss of functionality" without using the uprobabilistic indexes in some discrete spaces from sub - multitude in "sigma- algebra" (Gipich et al., 2013). It is shown that TP and PA (NASA) "sit" on probability, but TSS is "packed" in a Bole lattice with passing to the "unclear" vector lattice.

\section{References}

1. Advisory Circular (2006) AC № 120-92 (AFS-800) of FAA USA 06/22/06, \{AC 120-59A, AC 120-66 (ASAP), AC 120-79\}. MAK (11-12 October) 2006.

2. Airbus FAST (2005) (Technologies in SMS). EASA (December 2005) - www.airbusworld.com

3. Amer, Y. (2012) 10 Things You Should Know about SMS. FAA, Washington, 2012.

4. Austrian Standards: ONR 49000:2010 (2010) Risk Management for Organizations and Systems. ASI. 2010. pp. 12-21 (English).

5. ECAST (2012) Component of ESSI, European Strategic Safety Initiative. Guideline on the identification of danger. (Dynamics methods of Helicopter movement Simulation) EASA. 2012.

6. Everdij, M.H.C., Scholte, J.J., Zmarrou, H. (2013) General system of Risk evolution and Control, proposed by FAA with recommendation of NASA: NLR, NLR-CR-2011-110. May 2013.

7. Gipich, G.N., Kuklev, E.A., Evdokimov, V.G., Shapkin, V.S. (2013) Risks and safety of the aviation systems. Monograph. ISBN 978-5-903865-10-9. LTD. Publishing is a printed of "INSOFT". Moscow: Gos NII GA. 232 p.

8. IATA Safety Report (2014) ISBN 978-92-9252-582-8. Montreal-Geneva. 2015, 142 p.

9. ICAO Conference of higher level of Flight Safety (2010) (A37-WP/1P/1 - Appendix A, 13/7/10), Montreal (Canada).

10. International Programs. (2006) International programms PALM, TERASEC: www.aireurosafe.com. Preprint of MAK (iac) about Airbus Seminar for Civil Aviation Authorities in Moscow of CFIT, ALAR Tool kits - EASA, JAA (OAA). Moscow - MAK.

11. Khalkis, V. (2005) Main directions of Risk estimations in different Working spheres of activities. Riga, "SIA Jelgavas typography". Project in view of the directive EU 89/391/EEC. 2005. 74 p.

12. Malinowski, G.G., Kulba, V.V., Kosachenko, S.A., Shnirman, B. E. (2000) Management by a risk. Risk. Steady development. Synergy. Moscow: Science - "Series of cybernetic. Engineering", 431 p.

13. Levesohn, Nancy G. (2013) Engineering a Safer World. Systems Thinking. Applied to Safety. The MIT Press. Cambridge - Massachusetts. London, 2013.

14. Probabilistic Risk Assessment (PRA) (2002) Procedures Guide for NASA Managers and Practitioners - Office of Safety and Mission Assurance NASA. Washington, DC 20546 - August. 2002 (Version 1.1).

15. Report of Gos NII AS (2010) OOO 'Rinnicom” - V.G. Dudnikc, Moscow (in Russian).

16. Smurov, M.U., Kuklev, E.A., Evdokimov, V.G., Gipich, G.N. (2012) Safety of flights of civil aviation aircrafts taking in to account the risks of origin of negative events, Transport of Russian Federation. № 1 (38). SPb: 2012, p.54-58.

17. Standard of Main Aviation Risk estimation in SMS. (2012) Flight Safety Foundation - version 4, Airbus FAST (2005) (technologies in SMS). EASA (December 2005) - www.airbusworld.com.FAA apr. 2012.

18. The Little Oxford Thesaurus (2006) OUP Oxford: 3 edition. ISBN-10: 0198614497 